\title{
Necromasa de los bosques de Madre de Dios, Perú; una comparación entre bosques de tierra firme y de bajíos
}

\author{
Necromass in forests of Madre de Dios, Peru: a comparison between \\ terra firme and lowland forests
}

\author{
Alejandro Araujo-Murakami ${ }^{1}$, Alexander G. Parada ${ }^{1}$, Jeremy J. Terán ${ }^{2}$, Tim R. Baker ${ }^{3}$, \\ Ted R. Feldpausch ${ }^{3}$, Oliver L. Phillips ${ }^{3}$, Roel J.W. Brienen ${ }^{3 *}$
}

1 Museo de Historia Natural Noel Kempff Mercado, Casilla 2489, Av. Irala 565, Santa Cruz, Bolivia

2 Sociedad Boliviana de Historia Natural, Museo "Martin Cardenas H", Av Heroinas \# 0-0266, Cochabamba, Bolivia

3 Ecology and Global Change, School of Geography, University of Leeds, Leeds LS2 9JT, UK

*Autor para correspondencia Roel Brienen: r.brienen@leeds.ac.uk

Presentado:

Aceptado: 26/01/2011

Publicado online: 23/06/2011

\section{Resumen}

La cantidad de madera muerta o necromasa representa una importante porción de la biomasa y de los nutrientes en los bosques tropicales. Los objetivos de este estudio son: 1) hacer una evaluación y comparación entre la necromasa de los bosques de altura o tierra firme y los bosques inundables o bajíos, (2) estudiar las relaciones entre la necromasa, la biomasa aérea y la densidad de madera del bosque, y (3) proporcionar una primera estimación de la necromasa para todo el departamento de Madre de Dios. La necromasa gruesa y la masa aérea vegetativa fueron estudiados en tres diferentes lugares utilizando parcelas permanentes y líneas de intersección. El promedio del volumen de madera muerta gruesa fue de $72,9 \mathrm{~m}^{3} \mathrm{ha}^{-1}$, con un peso entre 24,8 y $30,7 \mathrm{Mg} \mathrm{ha}^{-1}$ dependiendo de la densidad de madera muerta usada en los cálculos. Los bosques de tierra firme contienen significativamente más madera muerta que los bosques inundables. La necromasa constituye $11 \%$ de la masa aérea vegetativa almacenada en los bosques de Madre de Dios. Finalmente, se estima que el departamento de Madre de Dios contiene alrededor de 100 mega toneladas de carbono en su madera muerta. Este valor es bastante alto, siendo diez veces más que la emisión anual de combustibles fósiles de Perú entre 2000 - 2008. Esta substancial porción de la necromasa enfatiza la importancia de estos tipos de estudios de campo, considerando que este componente de carbono en el bosque tropical no se logra detectar con otros métodos como la detección remota por satélites.

Palabras claves: Bosque tropical, madera muerta, Amazonía, almacén de carbono, ciclo de carbono.

\section{Abstract}

Stocks of dead wood or necromass represent an important portion of biomass and nutrients in tropical forests. The objectives of this study were: 1) to evaluate and compare the necromass of "terra firme" and lowlands forests, (2) to study the relationship between necromass, above-ground biomass and wood density, and (3) to estimate the necromass of the department of Madre de Dios, Peru. Stocks of necromass and above-ground biomass were estimated at three different locations using permanent plots and line intercept transects. The average volume of necromass for the three sites was $72.9 \mathrm{m3}$ ha-1 with an average weight varying between 24.8 and $30.7 \mathrm{Mg}$ ha-1, depending on the estimations of dead wood density used for the calculations. Terra firme forests had significantly higher stocks of necromass than lowland forests. The amount of necromass was $11 \%$ of the total above-ground biomass in Madre de Dios forests. The total stock of carbon stored in dead wood for the entire department of Madre de Dios was estimated to be approximately 100 mega tonnes of carbon. This is ten times more than the annual fossil fuel emissions of Peru between 2000 and 2008 . The substantial stocks of necromass emphasize the importance of these types of field studies, considering that this component of tropical forest carbon cannot be detected using other methods such as satellite remote sensing.

Keywords: Tropical forest, dead wood, Amazon, Carbon storage, Carbon cycle.

\section{Introducción}

La necromasa constituye una gran porción de la biomasa y de los nutrientes del ecosistema (Baker et al. 2007, Chao et al. 2009, Clark et al. 2002). Se estima que en los bosques de la Amazonía la necromasa constituye casi el 13\% de la biomasa aérea (Chao et al. 2009) y almacenan 9,6 Gt de carbono en toda la cuenca amazónica (Chao et al. 2009), lo cual es más que la emisión de carbón fósil causada por el ser humano durante el 2008 (Le Quere et al. 2009). Por lo tanto, la necromasa en los bosques tropicales es un componente importante en el ciclo global de carbono.

Las estimaciones de la necromasa en la Amazonía varían mucho desde casi ausente hasta más de $60 \mathrm{Mg} \mathrm{ha}^{-1}$, constituyendo hasta el 33\% de la biomasa aérea (Clark et al. 2002, Nascimento y Laurance 2002, Rice et al. 2004). La variación entre los estudios se debe en general a las diferencias entre los diferentes tipos de bosques y suelos. Considerando toda la cuenca amazónica, Chao et al. (2009) observaron que existe una gradiente en la necromasa que coincide con el gradiente de Este al Oeste en la biomasa aérea, la mortalidad y la densidad de madera (Baker et al. 2004, Malhi et al. 2004, 2006). Hay menos necromasa en el Oeste de la Amazonía que se correlaciona con menores cantidades de biomasa aérea, menores densidades de la madera, dinámica de bosque más elevada (Baker et al. 2004, Chao et al. 2009, Malhi et al. 2004, 2006), mayor fertilidad de suelo y una mala estructura física de suelo (Quesada et al. 2010a). Chao et al. (2009) observaron que la cantidad de necromasa depende de la densidad de madera, pero también de la cantidad de biomasa en la vegetación y de las tasas de mortalidad.

En pequeńas escalas espaciales también existen diferencias en la necromasa. En una comparación de diferentes tipos de bosques en el norte de Perú, por ejemplo, se observó que los bosques de tierra firme almacenan tres veces más necromasa que los bosques inundables (Chao et al. 2008). Aunque en los últimos años el número de estudios sobre la necromasa ha aumentado, todavía se entiende poco de los principales factores que la afectan y se requieren más estimaciones en los bosques tropicales.

En este estudio presentamos datos nuevos de las cantidades de necromasa en las cuencas del rió Los Amigos y rió Manu y sumados a los datos de Baker et al. (2007) para el río Tambopata, realizamos las primeras estimaciones de necromasa del Departamento de Madre de Dios (Perú), una particular región 
de la Amazonía central y este. Madre de Dios es el tercer departamento más grande de la Amazonía peruana y cubre un área de casi 85 mil kilometros cuadrados. En este departamento la Red Amazónica de Inventarios Forestales (RAINFOR (Peacock et al. 2007)), instaló parcelas permanentes de muestreo (PPM) en las cuencas del río Los Amigos, río Manu y río Tambopata con el objetivo de estudiar la dinámica de la biomasa y la biodiversidad a lo largo plazo.

Los objetivos de este estudio son: (1) hacer una evaluación y comparación entre la necromasa del bosque de altura o tierra firme y el bosque inundables o bajíos, que son los principales tipos de bosque en Madre de Dios, (2) estudiar si existe una relación entre la necromasa y la biomasa aérea y la densidad de madera del bosque y (3) proporcionar la primera estimación de la necromasa de todo el departamento de Madre de Dios, Perú.

\section{Material y métodos}

Sitios de estudio.- El trabajo de campo fue realizado en el departamento de Madre de Dios (Perú), en tres diferentes lugares: el Centro de Investigación y Capacitación Río Los Amigos (CICRA) (12³4'07”S; 7005’57”W), la estación Biológica Cocha Cashu del Parque Nacional Manu (PNM) (11 ${ }^{\circ} 53^{\prime} 8,27^{\prime}$ 'S; $\left.71^{\circ} 23^{\prime} 51,79^{\prime \prime W}\right)$ y en el Río Tambopata (1250'12,86"S; $\left.69^{\circ} 17^{\prime} 39,27^{\prime} \mathrm{W}\right)$ (Fig. 1). La precipitación anual varió poco entre los diferentes lugares con un promedio de $3034 \mathrm{~mm}$ para Cocha Cashu, 2417 mm para Río Tambopata (Malhi et al. 2004) y $2648 \mathrm{~mm}$ para los Amigos (CICRA 2010). La región tiene una estación seca de 3 meses entre junio y agosto con un promedio de lluvia mensual menor de $100 \mathrm{~mm}$ y una temperatura promedio anual de $24-25^{\circ} \mathrm{C}$.

Los sitios de estudio incluyen bosques inundables (Varzea) ó en sitios bajos, "bajíos" y bosques que nunca se inunda o "Tierra Firme”. Los orígenes de los suelos son Pleistoceno o Holoceno y están clasificados como ultisols (Quesada et al. 2010b). Los bosques de bajíos se encuentran detrás de las barreras ribereñas de los ríos de aguas blancas, donde frecuentemente el relieve desciende formando áreas anegables periódicamente. En los bosques de tierra firme el suelo está por encima del nivel máximo de las aguas, y nunca o muy raras veces pueden ser inundados. En los bosques de tierra firme los suelos pueden ser pedregosos, profundos y ricos en humus y mantienen su fertilidad mientras estén cubiertos con vegetación.

Métodos de campo.- En cada sitio se hizo un levantamiento de la cantidad de detritus de madera gruesa caída y en pie con diámetro mayor de $10 \mathrm{~cm}$. En este estudio se excluye la necromasa fina de madera con diámetros menores a $10 \mathrm{~cm}$, hojarasca, frutos, flores, etc.

En este estudio hemos utilizado dos métodos de muestreo de necromasa; i) en base a parcelas y ii) en base a líneas de intersección (van Wagner 1968). En las áreas de Manu y Los Amigos hemos levantado la cantidad de necromasa durante agosto del 2008 usando solamente el método en base a líneas de intersección, mientras que el levantamiento de cantidades de necromasa de Tambopata se realizó mediante una combinación de los dos métodos. Los datos de Tambopata ya han sido publicados en Baker et al. (2007), y fueron colectada entre abril y julio 2006.

Método en base a parcelas: El método en base a parcelas consiste simplemente de buscar en todo la parcela de 1 hectárea los árboles muertos. Este método fue solamente aplicado en Tambopata
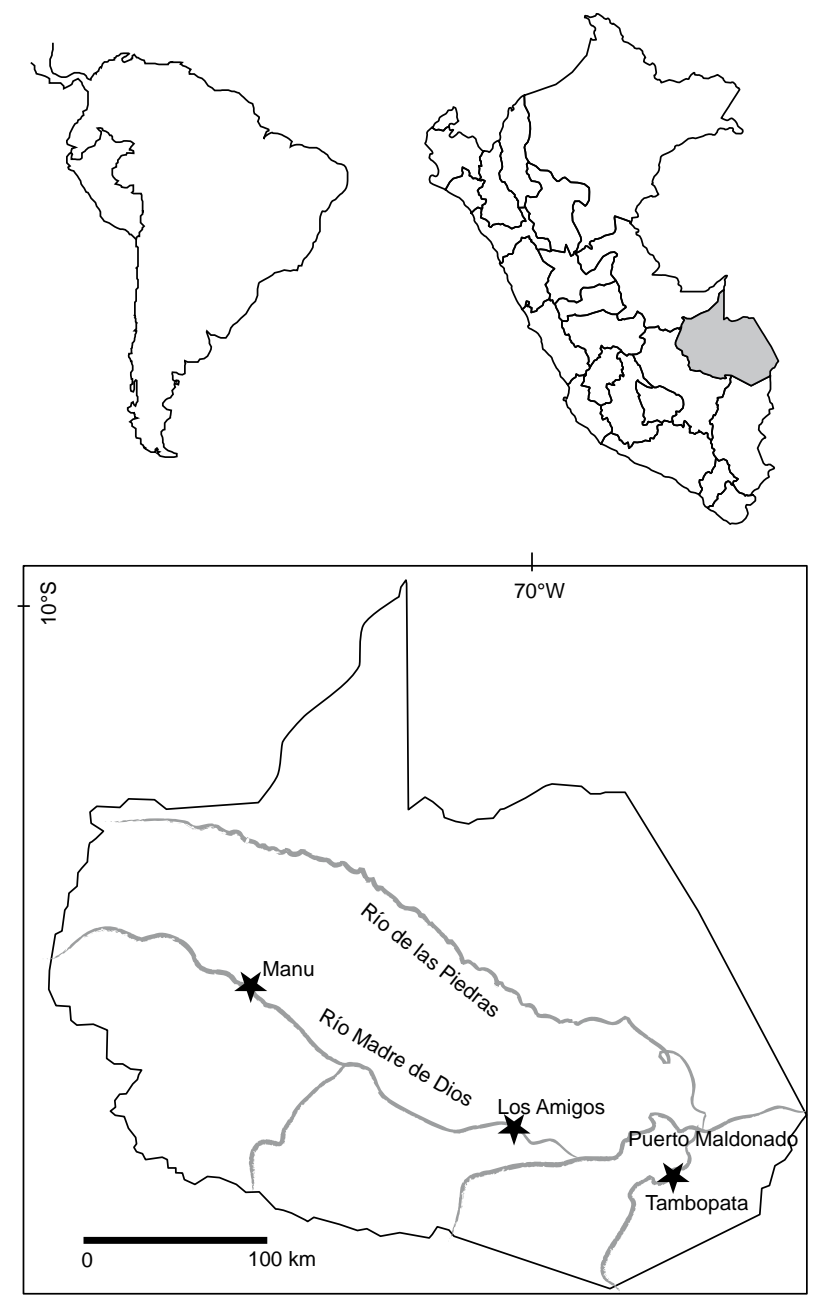

Figura 1. Ubicación del estudio indicando las áreas de estudio en el departamento de Madre de Dios, Perú.

para estimar la cantidad de necromasa de árboles muertos en pie y las cepas (ver Baker et al. (2007) para más detalle). Para los árboles muertos en el suelo usamos en Tambopata el método en base a líneas de intersección.

Método en base a líneas de intersección: Desde cada esquina de las parcelas de 100×100 m se trazaron líneas de intersección de $250 \mathrm{~m}$ de longitud lo cual constituye un total de $1000 \mathrm{~m}$ de línea por parcela. Cada una de las cuatro líneas parte desde una esquina de las parcelas y estaba orientada en dirección perpendicular a la otra línea, estando 100 metros de distanciada entre ellas para mantener la independencia. El método para las parcelas de Tambopata difirió un poco (ver Baker et al. 2007), así que cada línea fue de $100 \mathrm{~m}$ que resulta en $400 \mathrm{~m}$ en total para cada parcela (ver Tabla 1). En las cuatro líneas de $250 \mathrm{~m}$ se midieron todos los pedazos o trozos de madera muerta caída con diámetros menores o iguales a $10 \mathrm{~cm}$ que cruzaban la línea de intersección. Además, para estimar la cantidad de madera muerta en pie las líneas de $250 \mathrm{~m}$ se trasformaron en transectos de $10 \mathrm{~m}$ de ancho hacia los lados de las línea de intersección, donde fueron medidos todos los árboles muertos en pie y las cepas o tocones menores o iguales $10 \mathrm{~cm}$ de diámetro (DAP, Diámetro al Altura de Pecho, ó arriba de aletones).

Después de medir el diámetro interceptado en las líneas y el DAP en los transectos se anoto el estado de la descomposición de la madera muerta, que fue clasificada en un primer momento en cinco diferentes categorías en base a simples características 
Tabla 1. Características de los sitios de estudio y métodos de muestreo (línea y/o parcela)

\begin{tabular}{llccccccc}
\hline Región & Parcela & Código & Latitud & Longitud & Altura & $\begin{array}{c}\text { Tipo de } \\
\text { Bosque }\end{array}$ & $\begin{array}{c}\text { Método } \\
\text { Longitud de } \\
\text { línea (m) }\end{array}$ \\
\hline Los Amigos & Castañal & LAS-01 & $-12,5562$ & $-70,1068$ & 268 & Altura & línea & 1000 \\
Los Amigos & Jacaratia & LAS-02 & $-12,5333$ & $-70,0833$ & $\sim 240$ & Bajío & línea & 1000 \\
Manu, Cocha Cashu & Tierra Firme, Terraza & MNU-03 & $-11,9014$ & $-71,4014$ & 312 & Altura & línea & 1000 \\
Manu, Cocha Cashu & Tierra Firme Ravine & MNU-04 & $-11,9054$ & $-71,4016$ & 312 & Altura & línea & 1000 \\
Manu, Cocha Cashu & Trail 12 & MNU-05 & $-11,8789$ & $-71,4082$ & 312 & Bajío & línea & 1000 \\
Manu, Cocha Cashu & Trail 2 \& 31 & MNU-06 & $-11,8864$ & $-71,3792$ & 312 & Bajío & línea & 1000 \\
Río Tambopata & Parcela 0 & TAM 01 & $-12,8444$ & $-69,2891$ & 196 & Bajío & línea / parcela & 400 \\
Río Tambopata & Parcela 1 & TAM 02 & $-12,8348$ & $-69,2861$ & 210 & Bajío & línea parcela ${ }^{1}$ & 400 \\
Río Tambopata & Parcela 3 & TAM 05 & $-12,8299$ & $-69,271$ & 207 & Altura & línea parcela ${ }^{1}$ & 400 \\
Río Tambopata & Parcela 6 & TAM 07 & $-12,8252$ & $-69,2611$ & 220 & Altura & línea /parcela & 400 \\
Río Tambopata & Parcela 7 & TAM 08 & $-12,8269$ & $-69,2698$ & 224 & Altura & línea /parcela ${ }^{1}$ & 400 \\
\hline
\end{tabular}

${ }^{1}$ El método de línea de intersección fue aplicada para estimar el volumen de madera muerta en el suelo, y el método de parcela para estimar la madera muerta parada (ver Baker et al. 2007).

de la madera muerta (Clark et al. 2002, Delaney et al. 1998, Keller et al. 2004).

En este estudio se clasificó el estado de la descomposición de cada pieza de necromasa en una de las siguientes 5 clases: clase 1 corresponde a madera sólida, caída recientemente, con corteza intacta y ramas finas todavía adjuntas; clase 2 corresponde a madera sólida, pero sin ramas finas y con corteza que empieza a desprenderse; clase 3 corresponde a madera no sólida, en condiciones pobres, pero donde resultó difícil empujar una clavo dentro de la madera con la mano; clase 4 corresponde a madera blanda, madera podrida, donde un clavo podría ser empujado dentro de la madera fácilmente; clase 5 corresponde a madera suave, madera podrida que se rompe con facilidad al pisarla.

Varios estudios indican que las diferencias en la densidad de madera entre las clases de descomposición son pequeñas (Chao et al. 2008) o no significativas (Palace et al. 2008), y para facilitar el análisis y poder calcular las densidades según las formulas de Chao et al. (2009), las 5 clases obtenidas en un primer momento fueron agrupadas en 3 clases: (I) juntando las clases 1 con la clase 2, (II) la clase 3 y (III) juntando la clase 4 con la clase 5 .

\section{Cálculos de volumen de la madera en el suelo}

Para todas las piezas de madera en el suelo se calculó el volumen según:

$$
V=\left(\pi^{2} \times \Sigma d_{i}^{2}\right) / 8 L
$$

Donde $V$ es el volumen por unidad de área, $d_{i}$ es el diámetro (en $\mathrm{cm}$ ) del tronco $i$ y $L(\mathrm{~m})$ es el longitud de la línea-transecto (van Wagner 1968).

Para el cálculo del volumen de madera en pie se utilizó la formula de Chambers et al. (2001) de volumen de troncos, que se expresa:

$$
V=0,0011 D_{b}^{1,8516 *} H^{0,9053}\left(\left(2 H^{0,118}\right)-H^{0,118}\right) \text {, }
$$

Donde $D_{b}$ es el diámetro a la altura de pecho, y $H$ es la altura del tronco hasta el punto más alto donde tiene $10 \mathrm{~cm}$ de diámetro.

\section{Densidad de las clases de descomposición}

Los valores de densidades de las clases de descomposición $\left(\rho_{\mathrm{d}}, \mathrm{g} \mathrm{cm}^{-3}\right)$ son especifico de cada sitio, pero están altamente relacionado con la densidad de madera de los árboles vivos a nivel de parcela (Chao et al. 2008). Así, la densidad $\left(\rho_{d}\right)$ fue estimada como una función de la densidad promedia de los árboles vivos de la parcela, según la ecuación de Chao et al. (2008), que se expresa:

$$
\begin{aligned}
& \rho_{\mathrm{d}=1}=1,17\left[\rho_{\mathrm{BAj}}\right]-0,21 \\
& \mathrm{y} \\
& \rho_{\mathrm{d}=2}=1,17\left[\rho_{\mathrm{BA}}\right]-0,31
\end{aligned}
$$

Donde $\rho_{\mathrm{d}}=1$ y $\rho_{\mathrm{d}}=2$ representan las densidades de necromasa en clases de deterioro (d) I y II respectivamente, y $\rho_{\mathrm{BAj}}$ (g $\mathrm{cm}^{-3}$ ) es la densidad de la madera de árboles vivos de la parcela $j$. Para la necromasa en clase de deterioro III, se utilizó el valor medio de la densidad por detritus en "clase de deterioro tres" de estudios publicados de bosques neotropicales húmedos de las tierras bajas $\left(0,29 \mathrm{~g} \mathrm{~cm}^{-3}\right)$, como sugirió Chao et al. (2008).

Las densidades de madera de árboles vivos se obtuvo de la base de datos de RAINFOR (Peacock et al. 2007) y la base de datos de densidad de madera de especies de Baker et al. (2004) y Chave et al. (2006). La densidad de la madera se relacionó a los datos de parcelas en función a cada individuo o árbol, es decir se utiliza la densidad de la especie a la que corresponde cada individuo. Es así, que en los casos que faltaban datos de densidad de especies se utilizó el promedio de densidad por género o por familia. Para los árboles no identificados se utilizó el promedio de la densidad de madera de todos los árboles en la parcela.

La biomasa aérea gruesa (AGW, $\mathrm{Mg} \mathrm{ha}^{-1}$ ) fue estimada para cada parcela, utilizando las ecuaciones de Chambers et al. (2001), ajustadas por Baker et al. (2004) que incorpora la densidad de madera en este modelo. Todos los árboles menores o iguales 10 $\mathrm{cm}$ de diámetro fueron medidos en 2007 y 2008 a la altura de pecho (DAP; 1,30 m) ó para árboles con aletones por arriba de estos. Mayores detalles de los métodos de mediciones de diámetros se encuentra en el protocolo de RAINFOR (accesible en línea web: http://www.geog.leeds.ac.uk/projects/rainfor/pages/ manuals_eng.html).

\section{Estimaciones de la madera muerta en Madre de Dios}

Para estimar el total de la necromasa del departamento de Madre de Dios se utilizó el promedio de necromasa por hectárea multiplicado con el total de área de bosque de Madre de Dios. El departamento de Madre de Dios tiene una extensión de 8,476 millones de hectáreas (FAO 2005), de los cuales se estima que por lo menos $80 \%$ constituye bosque tropical. 


\section{Resultados}

Necromasa en los diferentes tipos de bosque.- El estudio presenta un promedio de $72,9 \mathrm{~m}^{3} \mathrm{ha}^{-1}$ de madera muerta, el cual varía desde 54,8 y $103,9 \mathrm{~m}^{3} \mathrm{ha}^{-1}$. El bosque de tierra firma o altura presenta volumen promedio $83,3 \mathrm{~m}^{3} \mathrm{ha}^{-1} \mathrm{y}$ el bosque inundable o de bajío presenta en promedio $60,4 \mathrm{~m}^{3} \mathrm{ha}^{-1}$ de madera muerta (Tabla 2). Es así, que los bosques de tierra firme contienen significativamente más volumen de madera muerta que los bosques de bajío (Mann-Whitney $\mathrm{U}, p<0,05$ ).

La necromasa, o el peso del volumen de madera muerta calculado en base de las densidades de madera de las diferentes clases de descomposición (ver métodos), tiene un promedio variable entre 30,7 y 24,8 $\mathrm{Mg} \mathrm{ha}^{-1}$, el primero basado en la densidad de madera muerta de un estudio realizado en Tambopata (Baker et al. 2007) y el segundo basado en la densidad de árboles vivos de cada parcela y las ecuaciones de Chao et al (2009). En ambos cálculos o estimaciones existe una diferencia significativa (MannWhitney $\mathrm{U}, p<0,05$ ) entre la necromasa de los bosques de tierra firme o altura y el bosque inundable o bajío. Siendo mayor el valor de necromasa en los bosques de tierra firme (Fig. 2).

La proporción (el ratio) promedio entre la necromasa parada y la del suelo (p/s) es de $25 \%$ pero varia bastante entre los diferentes sitios $(15-53 \%)$. La necromasa constituye $11 \%$ de la masa aérea vegetativa almacenado en las parcelas (Tabla 2).

La necromasa cuantificada en base a las ecuaciones de Chao et al. (2008), no está relacionado significativamente con la cantidad de biomasa en la vegetación aérea (AGB), pero si tiene una correlación significativa con la densidad de madera viva por parcela $(\rho j$, Pearson-r $=0,60 ; p<0,05)$.

\section{Proyecciones de la necromasa en Madre de Dios}

Usando los resultados de necromasa, se proyecto que el total de necromasa en el departamento de Madre de Dios está entre de 160 y $200 \mathrm{Mt}\left(=10^{12} \mathrm{~g}\right)$, variando según el tipo de cálculo de densidad de madera usado (ver métodos). Asumiéndose que casi el 50\% de la biomasa y necromasa es carbono (Elias y Potvin 2003), se estima que los bosques de Madre de Dios contienen

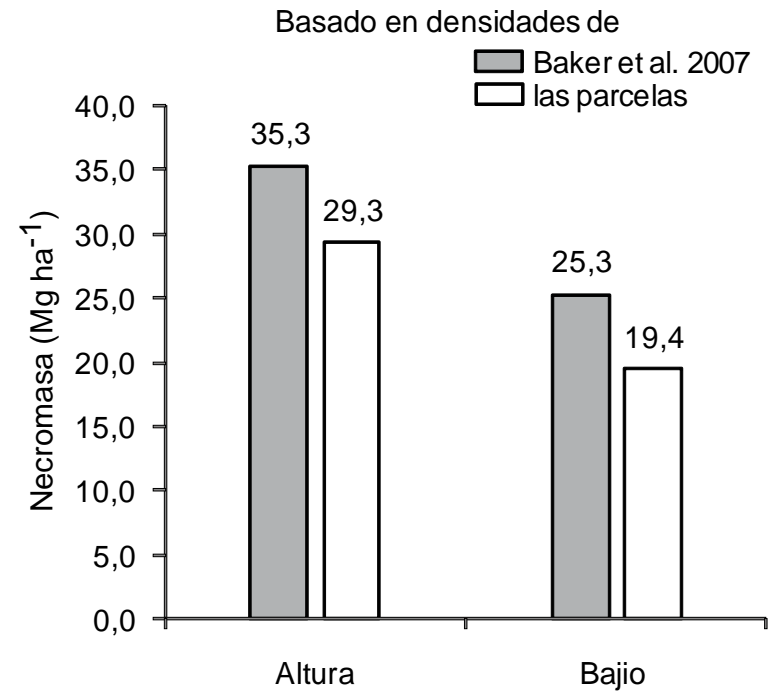

Figura 2. Promedios de necromasa en los bosques de altura o tierra firme y bosques de bajío o inundables, según los diferentes métodos de cálculo de necromasa (ver métodos).

entre 80 y $100 \mathrm{Mt}$ de carbono en la necromasa, dependiendo de qué valores de densidades de madera se usan para los cálculos. Junto con el carbono que se encuentra en la biomasa aérea (790 Mt) esto constituye un total de 870 - $890 \mathrm{Mt}$ de carbono aérea (nótense que esto no incluye el carbono contenido en las raíces y el suelo).

\section{Discusión}

Los valores observados en este estudio son casi el doble de los valores que Chao et al. (2009) reportó para el Suroeste de la Amazonía (17,5 $\left.\mathrm{Mg} \mathrm{ha}^{-1}\right)$, pero similar a los valores de necromasa de la misma región reportado por Baker et al. (2007), usando el método de la línea de intersección. De acuerdo con las observaciones de Baker et al. (2007), nuestros valores son relativamente bajos en comparación con la mayoría de los estudios de necromasa en bosques tropicales, con un valor extremo de $86,6 \mathrm{Mg} \mathrm{ha}^{-1}$ reportado para Tapajos en el Este de Amazonía (Rice et al. 2004).

Tabla 2. Necromasa y biomasa en los 11 sitios en el departamento de Madre de Dios. Donde, AGB, Biomasa Aérea Gruesa (Mg ha-1); $\rho_{\text {. }}$ promedio de densidad de madera de parcela j $\left(\mathrm{g} \mathrm{cm}^{-1}\right)$. V $\left(\mathrm{m}^{3} \mathrm{ha}^{-1}\right), \mathrm{N}$, necromasa $\left(\mathrm{Mg} \mathrm{ha}^{-1}\right)$ usando los valores de densidad de madera de Baker et al. $2007\left(\mathrm{~N}^{1}\right)$ o en base del promedio de densidad de madera por parcela $\left(\mathrm{N}^{2}\right.$, cf. Chao et la. 2009), p/s= relación (ratio) entre la necromasa parada y la necromasa caído en el suelo, N/AGB= relación (ratio) entre la necromasa y la biomasa.

\begin{tabular}{|c|c|c|c|c|c|c|c|c|c|c|c|}
\hline $\begin{array}{l}\text { Tipo de } \\
\text { Bosque }\end{array}$ & Código & AGB & рj & $\begin{array}{l}\text { Volumen } \\
\text { en pie }\end{array}$ & $\begin{array}{l}\text { Volumen } \\
\text { en el suelo }\end{array}$ & Total & $\mathbf{N}^{1)}$ & $\mathbf{N}^{2)}$ & $\mathrm{p} / \mathrm{s}$ & $\begin{array}{c}\text { N/ } \\
\text { AGB }\end{array}$ & Fuente \\
\hline Altura & LAS-01 & 248,4 & 0,55 & 55,0 & 48,9 & 103,9 & 46,5 & 37,7 & $53 \%$ & $15 \%$ & Este estudio \\
\hline Altura & MNU-03 & 226,5 & 0,51 & 21,0 & 53,9 & 74,9 & 32,4 & 23,6 & $28 \%$ & $10 \%$ & Este estudio \\
\hline Altura & MNU-04 & 230,3 & 0,55 & 21,0 & 42,9 & 63,9 & 27,4 & 21,8 & $33 \%$ & $9 \%$ & Este estudio \\
\hline Altura & TAM 05 & 215,2 & 0,61 & 9,9 & 56,2 & 66,1 & 27,8 & 25,6 & $15 \%$ & $12 \%$ & Baker et al. (2007) \\
\hline Altura & TAM 07 & 223,6 & 0,58 & 16,2 & 85,3 & 101,6 & 39,7 & 33,1 & $16 \%$ & $15 \%$ & Baker et al. (2007) \\
\hline Altura & TAM 08 & 188,8 & 0,60 & 16,1 & 73,3 & 89,4 & 37,8 & 34,0 & $18 \%$ & $18 \%$ & Baker et al. (2007) \\
\hline Promedio & & 222,1 & 0,56 & 22,5 & 60,8 & 83,3 & 35,3 & 29,3 & $27 \%$ & $13 \%$ & \\
\hline Bajío & LAS-02 & 240,2 & 0,52 & 14,5 & 41,4 & 55,9 & 24,2 & 18,2 & $26 \%$ & $8 \%$ & Este estudio \\
\hline Bajío & MNU-05 & 299,5 & 0,54 & 14,9 & 47,0 & 61,9 & 25,9 & 20,3 & $24 \%$ & $7 \%$ & Este estudio \\
\hline Bajío & MNU-06 & 272,3 & 0,52 & 9,0 & 51,0 & 60,0 & 27,2 & 20,3 & $15 \%$ & $7 \%$ & Este estudio \\
\hline Bajío & TAM 01 & 201,9 & 0,53 & 15,9 & 53,2 & 69,1 & 28,0 & 21,5 & $23 \%$ & $11 \%$ & Baker et al.(2007) \\
\hline Bajío & TAM 02 & 210,6 & 0,54 & 11,0 & 43,8 & 54,8 & 21,3 & 16,8 & $20 \%$ & $8 \%$ & Baker et al. (2007) \\
\hline Promedio & & 244,9 & 0,53 & 55,0 & 48,8 & 60,4 & 25,3 & 19,4 & $22 \%$ & $8 \%$ & \\
\hline
\end{tabular}

1) En base a valores de densidades de Baker et al. (2007); ${ }^{2}$ En base a valores de densidades de ecuaciones de Chao et al. 2009 y valores de densidad de madera de las parcelas 
Los niveles de necromasa reportados en este estudio concuerdan con el gradiente observado por Chao et al. (2009), quienes anotaron que las regiones del sur y el oeste de la Amazonía tienen valores más bajos de necromasa en comparación con el este y noreste de la Amazonía (Chao et al. 2009). Estos bajos niveles de necromasa coinciden con valores bajos de biomasa y densidad de madera (Baker et al. 2004), una alta productividad de madera (Baker et al. 2004), y altas tasas de mortalidad, y esto demuestra que en estas áreas de la Amazonía el reciclaje de carbono parece ser más elevado. Este carácter distinto de la dinámica del bosque de esta parte de la Amazonía es probablemente en gran parte causado por las diferencias en las propiedades químicas y físicas del suelo entre las regiones. Quesada et al. (2010) demostró que el sur y el oeste de la Amazonía tienen suelos de propiedades menos favorables que podrían ser la causa de la mayor tasa de mortalidad, lo cual influye en la estructura del bosque y la productividad de madera. La elevada mortalidad forestal registrada en estos bosques da como resultado una mayor cantidad de luz en el bosque, y en conjunto con el alto contenido de nutrientes de los suelos de estos bosques del sur y del oeste de la Amazonía favorecen a los árboles con estrategia de crecimiento rápido, baja densidad de madera y corta vida (Quesada et al. 2010a). Las diferencias en composición de especies y densidades de madera entre los diferentes sectores (Baker et al. 2004) de la Amazonía pueden afectar las tasas de descomposición de madera y ser las principales causas de las diferencias en las cantidades de necromasa entre sectores.

A nivel regional, se encuentra una gran variación en la necromasa entre los diferentes sitios, lo cual concuerda con las observaciones de Chao et al. (2008), quienes señalan grandes diferencias entre diferentes tipos de bosque. En el presente estudio se encontró que en los bosques inundables o de bajío existe una menor cantidad de necromasa que en los bosques de tierra firme o altura. Esto puede deberse a las inundaciones frecuentes de los bosques de bajíos, donde puede suceder la reubicación de la madera muerta en el suelo en áreas más bajas, pero también podría deberse a las mayores tasas de la descomposición de madera muerta en los bosques de bajío por efecto del ciclo de mojado y secado de la madera (Martius 1997).

Concordando con el estudio de Chao et al. (2008), nosotros observamos que la necromasa está relacionado con la densidad de madera de árboles vivos; esta relación fue como se esperaba, las tasas de descomposición fueron más lentas en árboles con una densidad de madera más pesada (Chambers et al. 2000, Martius 1997). No se observó relación entre el volumen ó masa de madera muerta y la biomasa en la vegetación aérea (AGW) en este estudio. Chao et al. (2009) observó que al nivel de toda la Amazonía y para bosques de un solo tipo (tierra firme) existe una relación entre la necromasa y la biomasa. Esta ausencia de una relación entre la necromasa y la biomasa en nuestro trabajo podría deberse a que fueron comparados diferentes tipos de bosques en los que existen diferencias en la densidad de madera de los árboles vivos y tasas de mortalidad, que vienen a ser algunos de los factores que causan diferencias en la cantidad de madera muerta entre sitios.

Basados en el promedio de la necromasa del bosque inundable y bosque de tierra firme se estima que el Departamento de Madre de Dios contiene alrededor de 100 mega toneladas de carbono en su madera muerta. Este valor es bastante alto, siendo diez veces más que la emisión anual de combustibles fósiles de Perú entre 2000 - 2008 (8,8 Mt; Boden et al. 2010). Esta estimación es todavía conservadora, porque existen más áreas de bosque de altura y estos tienen los valores más altos de madera muerta. Además, la suposición de que solo el $80 \%$ de la superficie del departamento de Madre de Dios son bosques también es conservativa, porque en las áreas de aprovechamiento forestal la cantidad de madera muerta es temporalmente elevada y no es considerada (Feldpausch et al. 2005). Junto, con el carbono de la biomasa aérea, el contenido de carbono en los bosques de Madre de Dios es de 880 megatoneladas de carbono, o cien veces la emisión anual de combustibles fósiles de Perú.

El presente trabajo ofrece la primera estimación de necromasa en el departamento de Madre de Dios; aunque para una estimación más precisa se requiere un muestreo más intensivo, una gran cantidad de necromasa es determinada, lo cual enfatiza la importancia de estos estudios en la estimación de los diferentes componentes de carbono en los bosques tropicales, algo que no se logra conocer por la detección remota satelital. Especialmente para las estimaciones de la necromasa o el carbono en los suelos, estudios como los realizados en este trabajo son indispensables, a pesar de los recientes avances en detección remota de estimaciones de biomasa aérea (Chambers et al. 2007).

\section{Agradecimientos}

Agradecemos a la fundación de Betty y Gordon Moore por su apoyo al proyecto RAINFOR, y a los participantes en el taller RAINFOR de Los Amigos, Perú en 2008. También agradecemos a SERNANP/ INRENA por el permiso para la realización de esta investigación.

\section{Literatura citada}

Baker T.R., E.N.H. Coronado, O.L. Phillips, et al. 2007. Low stocks of coarse woody debris in a southwest Amazonian forest. Oecologia 152: 495-504

Baker T.R., O.L. Phillips, Y. Malhi, et al. 2004. Variation in wood density determines spatial patterns in Amazonian forest biomass. Global Change Biol. 10: 545-562

Boden T., G. Marland \& R.J. Andres. 2010. National CO2 Emissions from Fossil-Fuel Burning, Cement Manufacture, and Gas Flaring: 1751-2006. doi 10.3334/CDIAC/00001_V2010. Carbon Dioxide Information Analysis Center, Oak Ridge National Laboratory; Oak Ridge, Tennessee.

Chambers J.Q., G.P. Asner, D.C. Morton, et al. 2007. Regional ecosystem structure and function: ecological insights from remote sensing of tropical forests. Trends Ecol. Evol. 22: 414-423

Chambers J.Q., J. dos Santos, R.J. Ribeiro \& N. Higuchi. 2001. Tree damage, allometric relationships, and above-ground net primary production in central Amazon forest. For. Ecol. Manag. 152: 73-84

Chambers J.Q., N. Higuchi, J.P. Schimel, L.V. Ferreira \& J.M. Melack. 2000. Decomposition and carbon cycling of dead trees in tropical forests of the central Amazon. Oecologia 122: $380-388$

Chao K.J., O.L. Phillips \& T.R. Baker. 2008. Wood density and stocks of coarse woody debris in a northwestern Amazonian landscape. Canadian Journal of Forest Research-Revue Canadienne De Recherche Forestiere 38: 795-805

Chao K.J., O.L. Phillips, T.R. Baker, et al. 2009. After trees die: quantities and determinants of necromass across Amazonia. Biogeosciences Discuss., 6, 1979-2006 < http:// biogeosciences-discuss.net/6/1979/2009/bgd-6-19792009.pdf> (access 09/09/2010) 
Chave J., H.C. Muller-Landau, T.R. Baker, et al. 2006. Regional and phylogenetic variation of wood density across 2456 neotropical tree species. Ecol. Appl. 16: 2356-2367

CICRA. 2010. El Cicra en 60 segundos, <http://cicra.acca.org.pe/ espanol/cicra_60_segundos.html $>$ (access 09/09/2010)

Clark D.B., D.A. Clark, S. Brown, S.F. Oberbauer \& E. Veldkamp. 2002. Stocks and flows of coarse woody debris across a tropical rain forest nutrient and topography gradient. For. Ecol. Manag. 164: 237-248

Delaney M., S. Brown, A.E. Lugo, A. et al. 1998. The quantity and turnover of dead wood in permanent forest plots in six life zones of Venezuela. Biotropica 30: 2-11

Elias M. \& C. Potvin. 2003. Assessing inter- and intra-specific variation in trunk carbon concentration for 32 neotropical tree species. Can. J. For. Res. 33: 6

FAO. 2005. Microfinance and forest-based small-scale enterprises. FAO Forestry Paper No. 146. Rome.

Feldpausch T.R., S. Jirka, C.A.M. Passos, et al. 2005. When big trees fall: Damage and carbon export by reduced impact logging in southern Amazonia. For. Ecol. Manag. 219: 199-215

Keller M., M. Palace, G.P. Asner, et al. 2004. Coarse woody debris in undisturbed and logged forests in the eastern Brazilian Amazon. Global Change Biol. 10: 784-795

Le Quere C., M.R. Raupach, J.G. Canadell, et al. 2009. Trends in the sources and sinks of carbon dioxide. Nature Geoscience 2: 831-836

Malhi Y., T.R. Baker, O.L. Phillips, et al. 2004. The above-ground coarse wood productivity of 104 Neotropical forest plots. Global Change Biol. 10: 563-591
Malhi Y., D. Wood, T.R. Baker, et al. 2006. The regional variation of aboveground live biomass in old-growth Amazonian forests. Global Change Biol. 12: 1107-1138

Martius C. 1997. Decomposition of wood. In: Junk WJ (ed) The central Amazon floodplain- ecology of a pulsing system. Springer, Germany

Nascimento H.E.M. \& W.F. Laurance. 2002. Total aboveground biomass in central Amazonian rainforests: a landscapescale study. For. Ecol. Manag. 168: 311-321

Palace M., M. Keller \& H. Silva. 2008. Necromass production: Studies in undisturbed and logged Amazon forests. Ecol. Appl. 18: 873-884

Peacock J., T.R. Baker, S.L. Lewis, et al. 2007. The RAINFOR database: monitoring forest biomass and dynamics. J. Veg. Sci. 18: 535-542

Quesada C.A., J. Lloyd, M. Schwarz, et al. 2010a. Regional and large-scale patterns in Amazon forest structure and function are mediated by variations in soil physical and chemical properties. Biogeosciences Discuss., 6, 3993-4057< http:// biogeosciences-discuss.net/6/3993/2009/bgd-6-39932009.pdf $>$ (access 09/09/2010)

Quesada C.A., J. Lloyd, M. Schwarz, et al. 2010b. Variations in chemical and physical properties of Amazon forest soils in relation to their genesis. Biogeosciences Discuss., 6, 39233992 <http://biogeosciences-discuss.net/6/3923/2009/ bgd-6-3923-2009.pdf> (access 09/09/2010)

Rice A.H., E.H. Pyle, S.R. Saleska, L. Hutyra, M. Palace, M. Keller, P.B. de Camargo, K. Portilho, D.F. Marques \& S.C. Wofsy. 2004. Carbon balance and vegetation dynamics in an old-growth Amazonian forest. Ecol. Appl. 14: S55-S71

van Wagner C.E. 1968. The line intersect method in forest fuel sampling. For. Sci. 14: 20-26 